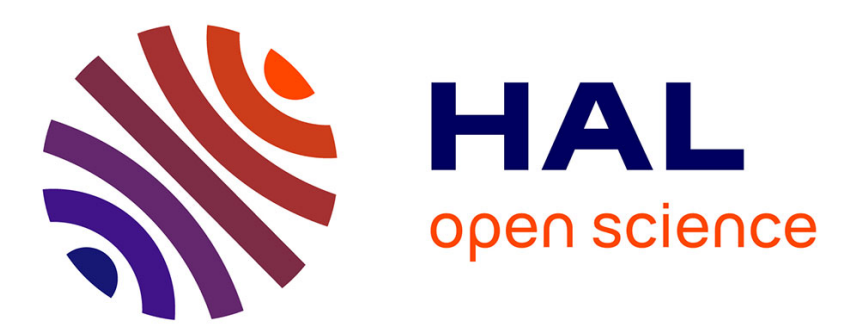

\title{
Distribution functions of a heteronuclear hard dumbbell at a hard wall. Pure fluid and mixtures with hard spheres
}

Tomas Boublik

\section{- To cite this version:}

Tomas Boublik. Distribution functions of a heteronuclear hard dumbbell at a hard wall. Pure fluid and mixtures with hard spheres. Molecular Physics, 2009, 107 (03), pp.205-211. 10.1080/00268970902766725 . hal-00513253

\section{HAL Id: hal-00513253 \\ https://hal.science/hal-00513253}

Submitted on 1 Sep 2010

HAL is a multi-disciplinary open access archive for the deposit and dissemination of scientific research documents, whether they are published or not. The documents may come from teaching and research institutions in France or abroad, or from public or private research centers.
L'archive ouverte pluridisciplinaire HAL, est destinée au dépôt et à la diffusion de documents scientifiques de niveau recherche, publiés ou non, émanant des établissements d'enseignement et de recherche français ou étrangers, des laboratoires publics ou privés. 


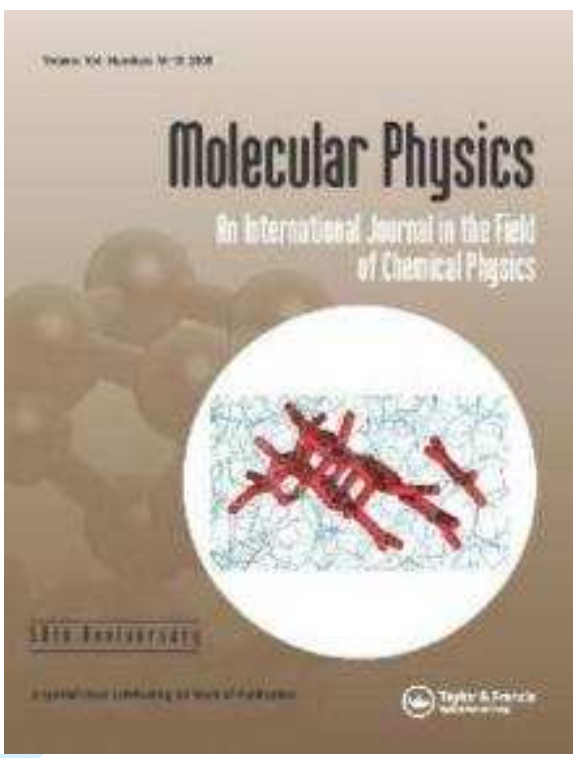

\section{Distribution functions of a heteronuclear hard dumbbell at a hard wall. Pure fluid and mixtures with hard spheres}

\begin{tabular}{|c|c|}
\hline Journal: & Molecular Physics \\
\hline Manuscript ID: & TMPH-2008-0405 \\
\hline Manuscript Type: & Full Paper \\
\hline $\begin{array}{r}\text { Date Submitted by the } \\
\text { Author: }\end{array}$ & 01-Dec-2008 \\
\hline Complete List of Authors: & $\begin{array}{l}\text { Boublik, Tomas; J.E.Purkinje Univ., Chemistry; Charles Univ., Phys. } \\
\text { \& Macromol. Phys. }\end{array}$ \\
\hline Keywords: & $\begin{array}{l}\text { Inhomogeneous system, Distribution function, Heteronuclear } \\
\text { Dumbbell, Residual chemical potential, Geometric quantities of } \\
\text { enlarged dumbbell }\end{array}$ \\
\hline \multicolumn{2}{|c|}{$\begin{array}{l}\text { Note: The following files were submitted by the author for peer review, but cannot be converted } \\
\text { to PDF. You must view these files (e.g. movies) online. }\end{array}$} \\
\hline HETHD.tex & \\
\hline
\end{tabular}

\section{S) ScholaroNE \\ Manuscript Central}




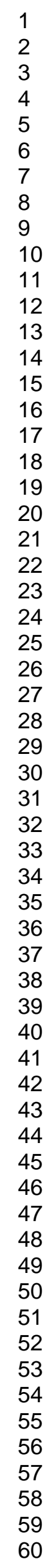

URL: http://mc.manuscriptcentral.com/tandf/tmph 


\section{Distribution functions of a heteronuclear hard dumbbell at a hard wall. Pure fluid and mix- tures with hard spheres}

Tomáš Boublık

Department of Chemistry, J.E. Purkinje University, 40096 Ústi nad Labem,

Czech Republic

e-mail: boublikt@ujep.cz 
Distribution functions (angle-averaged site/wall correlation functions) in the inhomogeneous systems of a heteronuclear hard dumbbell (of the site diameter ratio $\sigma_{2} / \sigma_{1}=0.5$ and $L^{*}=0.625$ ) were studied theoretically by applying a simple method to determine the background (cavity) correlation function from the residual chemical potentials of the hard dumbbell, hard wall and the corresponding combined body. Pure fluid and approximately equimolar mixtures of dumbbells with hard spheres were considered at several densities. Theoretical distribution functions for the most important site/wall distances were calculated and compared with simulation data; fair agreement was found. Advantage of the proposed method is its simplicity (the residual chemical potential depends, besides the packing fraction, only on the geometric characteristics of the hard body - its volume, surface area and mean radius); the method provides quick information on the density profiles in different inhomogeneous systems.

Key words: Inhomogeneous system, Distribution function, Heteronuclear Dumbbell, Cavity function, Residual chemical potential, Geometric quantities 


\section{Introduction}

Knowledge of the density profiles in the inhomogeneous systems of hard bodies represents an important input for theories of adsorption, catalysis or behavior of nano-systems. The problem of determining the density profiles or distribution functions has been tackled by simulation technique [1]-[6] or theoretically by employing the solution of the modified Orstein-Zernike equation [7]-[8] or by applying the density functional theory [9]-[11]. Solution of both types of theoretical expressions have been obtained by different numerical methods; some input data (for the corresponding homogeneous systems) are used.

An alternative to these rather involved approaches represents a method, based on the determination of the background (cavity) correlation function (bcf) from the residual chemical potential of a pair of interacting hard bodies and of the corresponding "combined body" (CB), resulting from the overlap of the particles of the considered pair. The formulation of the method was given by Meeron and Siegert[12] and further developed by Ballance and Speedy[13], Speedy[14], Labík et al. [15]-[16] and the present author [17][18]. In the original approaches, dealing with the hard sphere (hs) system, the characteristic geometric quantities - volume, $V$, surface area, $S$, mean radius, $R$ (mean curvature integral, divided by $4 \pi$ ), for the dumbbell with the site-site distances larger than a sum of radii of the both interacting hs's were obtained as a prolongation of formulas for $V$ and $S$ holding for the shorter site-site distances; in the case of $R$, the value calculated for the corresponding convex body, enveloping the given hard dumbbell (hd) was used. Such approximations yielded very precise values of the distribution functions for distances slightly larger than the contact one, but diverge at larger hs's distances.

This fact and difficulties with the definition of the combined body in the case of inhomogeneous systems led us to the introduction of a new CB - the enlarged hard dumbbell (ehd), i.e. a hard body originated when the probe hard sphere is rolled in contact with the sites of the original hd [19]. This new model of CB makes it possible to determine bcf in the most important range of hb distances and determine bcf of inhomogeneous systems.

The approach was recently applied to inhomogeneous systems of hs near a planar hard wall (hw), hs in a hard pore, homonuclear hb/wall systems with $\mathrm{hb}$ in the parallel and perpendicular orientations (with respect to the wall plane), full site-wall distribution function and to ternary hs systems with hs's differing considerably in their size [19]-[22]. It was found that within 
the considered approach, rdf's for different pairs of components might be easily calculated just by modifying the probe hard sphere radius and the non-sphericity parameters of the hs system under consideration (characterized by the composition of different hs's,).

In this contribution we study an application of the simple method to determine the site/wall distribution functions in the pure heteronuclear dumbbell system and in the hhd-hs mixtures. In the first part of the paper a brief outline of the method to determine the background correlation function is given and the way of determining the geometric characteristics of different ehd/wall pairs is summarized. In the next section the details of the determination of geometric quantities of the heteronuclear dumbbell and of the corresponding combined hard bodies are discussed. Next part brings a comparison of the calculated distribution functions with the simulation results (taken from figures of the original paper [23]). Conclusions summarize the main traits of the approach and possible ways of further improvement. 


\section{Theory}

The present theory is grounded on the relation between the background (cavity) correlation function[12] (bcf) $Y$,

$$
Y(x)=\exp \left[u(x) / k_{B} T\right] g(x)
$$

(where $g$ is the pair distribution function, $u$ - pair potential, $\mathrm{x}$ - distance) and the residual chemical potentials of a pair of interacting hard molecules and of the "combined body" (CB) (originated by overlapping of the given molecular pair), infinitely diluted in a system containing both the molecules $i, j$. For $Y$ it holds true

$$
k_{B} T \ln Y(x)=\Delta \mu_{i}+\Delta \mu_{j}-\Delta \mu^{C B}(x)
$$

(where $T$ - temperature and $k_{B}$ - Boltzmann constant). In the case of hard bodies and distances $x$ larger than the contact one, the pair potential $u(x)=0$ and bcf equals to the correlation function, $g(x)$.

The residual chemical potential of hard spheres [or non-spherical convex hard bodies (hb)] can be obtained as a derivative of the residual Helmholtz function, $\Delta A$, which is a function of the packing fraction, $y$, and nonsphericity parameters. For the fused hard sphere model or ehd's, no general/exact expression stands at our disposal; thus, expressions derived for $\Delta A$ of hard convex body systems have to be used.

The choice of the expression for the residual Helmholtz function depends on the system under consideration. In the case of the inhomogeneous systems, which are related to the colloidal limit of the hard body mixture, the adequate choice of the expression for $\Delta A$ is that, which meets the self-consistent constrains (SC) (see Ref [24]-[25]). The following equation meets fairly well the SC conditions and represents thus the proper choice of the relation for $\Delta A$ (for a pure hb fluid)

$$
\frac{\Delta A}{N k_{B} T}=-\ln (1-y)+\frac{3 \alpha y}{(1-y)}+\frac{\beta y^{2}(3 / 2-5 y / 12)}{(1-y)^{2}}
$$

Parameters of non-sphericity, $\alpha$ and $\beta$ for non-spherical hard body systems are defined as

$$
\alpha_{i}=R_{i} S_{i} / 3 V_{i} \quad \beta_{i}=Q_{i} S_{i}^{2} / 9 V_{i}^{2}
$$


where $V_{i}, S_{i}, R_{i}$ and $Q_{i}$ are the hcb geometric characteristics - volume, surface area, mean radius (exactly the mean curvature integral, divided by $4 \pi$ ); $Q$ is a quantity of dimension $\left[1^{2}\right]$, for which inequality $S_{i} / 4 \pi \leq Q \leq R_{i}^{2}$ holds true.

For mixtures of components $k=i, j \ldots$, the non-sphericity parameters are

$$
\alpha_{s}=r s / 3 \rho v \quad \beta_{s}=q s^{2} / 9 \rho v^{2}
$$

where $r=\rho \Sigma x_{k} R_{k}, s=\rho \Sigma x_{k} S_{k}, q=\rho \Sigma x_{k} Q_{k}$ and $v=y=\rho \Sigma x_{k} V_{k}$ and the extended expression, for the residual chemical potential, corresponding to (1) reads as

$$
\begin{gathered}
\beta \Delta \mu=-\ln (1-y)+\frac{y}{(1-y)}\left[3 \alpha\left(R^{*}+S^{*}\right)+V^{*}\right]+ \\
\frac{y^{2}}{(1-y)^{2}}\left[\beta\left(Q^{*}+2 S^{*}\right)(3 / 2-y / 3)+3 \alpha V^{*}\right]+\frac{y^{3}}{(1-y)^{3}}(7 / 3-y / 6) \beta V^{*}
\end{gathered}
$$

Here $R^{*}, S^{*}, V^{*}, Q^{*}$ are dimensionless quantities, $R^{*}=R / R_{\text {ref }}, S^{*}=S / S_{\text {ref }}$, $V^{*}=V / V_{\text {ref }}, Q^{*}=Q / Q_{\text {ref }}$. The reference quantities in the case of pure fluids are those of the considered system, thus $R_{i}^{*}, S_{i}^{*}, V_{i}^{*}, Q_{i}^{*}$, equal to one, whereas $R^{C B *}=R^{C B} / R_{i}, S^{C B *}=S^{C B} / S_{i}$, etc. For mixtures of components $k=i, j . . R_{\text {ref }}=\Sigma x_{k} R_{k}$, generally $X_{\text {ref }}=\sum x_{i} X_{i}$, where $x_{i}$ denotes here the mole fraction of component $i$. Defining

$$
\begin{aligned}
\Delta r=R_{i}^{*}+R_{j}^{*}-R^{C B *}, \quad \Delta s & =S_{i}^{*}+S_{j}^{*}-S^{C B *}, \quad \Delta v=V_{i}^{*}+V_{j}^{*}-V^{C B *} \\
\Delta q & =Q_{i}^{*}+Q_{j}^{*}-Q^{C B *}
\end{aligned}
$$

one can write

$$
\begin{gathered}
\ln Y=-\ln (1-y)+\frac{y}{(1-y)}[3 \alpha(\Delta r+\Delta s)+\Delta v]+ \\
\frac{y^{2}}{(1-y)^{2}}[\beta(\Delta q+2 \Delta s)(3 / 2-y / 3)+3 \alpha \Delta v]+\frac{y^{3}}{(1-y)^{3}}(7 / 3-y / 6) \beta \Delta v
\end{gathered}
$$

\subsection{Pure hard dumbbells}

As mentioned before, eq (4) was derived for hard convex bodies. In order to describe the behavior of non-convex bodies - diatomics, (triatomics etc.) and combined bodies - we adopt a model of the enlarged hard dumbbell 
(ehd) (generally enlarged fused hard body), originates when the probe hard sphere is rolled over hard dumbbell (in general case over a fused hard sphere body). The enlarged hard body possesses a smooth surface area (without any discontinuities and cusps). The idea of ehd is especially helpful in the case of the description of inhomogeneous systems of hard bodies near a hard wall.

In order to determine the geometric quantities of ehd, we employed the method due to Connolly [26, 27], in which the ehd surface area and volume were obtained as sums of the convex and saddle contributions (no concave part was present). For the heteronuclear hard dumbbell, studied in this work, with the site-site ratio $p=\sigma_{2} / \sigma_{1}=0.5$, the reduced site-site distance $L^{*}=l / \sigma_{1}=0.625$ (and radii $r_{i}=\sigma_{i} / 2$ ), the ehd surface area is given by two convex and two saddle contributions, see Figure 1 . The surface area of the studied ehd is

$$
S_{1}=2 \pi r_{1}^{2}\left(1+\sin \theta_{1}\right)+2 \pi r_{2}^{2}\left(1+\sin \theta_{2}\right)+2 \pi r_{p}\left[X\left(\theta_{1}+\theta_{2}\right)-r_{p}\left(\sin \theta_{1}+\sin \theta_{2}\right)\right]
$$

Here $r_{p}$ is the probe radius, $X=\left(r_{1}+r_{p}\right) \cos \theta_{1}$ and $\theta_{i}=\arcsin \left[d_{i} /\left(r_{i}+r_{p}\right)\right]$. Then

$$
\begin{gathered}
S_{1} / \pi \sigma_{1}^{2}=\left[\left(1+\sin \theta_{1}\right)+p^{2}\left(1+\sin \theta_{2}\right)+\right. \\
\left.r_{p}^{*}\left(1+r_{p}^{*}\right)\left(\theta_{1}+\theta_{2}\right) \cos \theta_{1}-r_{p}^{* 2}\left(\sin \theta_{1}+\sin \theta_{2}\right)\right] / 2
\end{gathered}
$$

(where $r_{p}^{*}=r_{p} / r_{1}$ ). The ehd volume of the considered dumbbell is similarly

$$
\begin{aligned}
& V_{1} /\left(\pi \sigma_{1}^{3} / 6\right)=\frac{1}{2}\left[\left(1+\sin \theta_{1}+\cos ^{2} \theta_{1} \sin \theta_{1} / 2\right)+p^{3}\left(1+\sin \theta_{2}+\cos ^{2} \theta_{2} \sin \theta_{2} / 2\right)\right] \\
& +\frac{3}{4} r_{p}^{*}\left(1+r_{p}^{*}\right) \cos \theta_{1}\left[\left(1+r_{p}^{*}\right) \cos \theta_{1}\left(\sin \theta_{1}+\sin \theta_{2}\right)-r_{p}^{*}\left(\cos \theta_{1}+\cos \theta_{2}\right)\right] \\
& +\frac{r_{p}^{* 3}}{4}\left(\cos ^{2} \theta_{1} \sin \theta_{1}+\cos ^{2} \theta_{2} \sin \theta_{2}+2 \sin \theta_{1}+2 \sin \theta_{2}\right)-\frac{3}{4} r_{p}^{* 2}\left(1+r_{p}^{*}\right) \cos \theta_{1}\left(\theta_{1}+\theta_{2}\right)
\end{aligned}
$$

The mean radius is given by sums of the convex and saddle contributions, obtained from eq (5) by differentiation with respect to $r_{1}$ resp. $r_{2}$ and minus derivative of the saddle parts of surface areas with respect to $r_{p}$; thus

$$
R_{1} / r_{1}=\frac{1}{2}\left[\left(1+\sin \theta_{1}\right)+p\left(1+\sin \theta_{2}\right)+r_{p}^{*}\left(\sin \theta_{1}+\sin \theta_{2}\right)-\left(1+r_{p}^{*}\right) \cos \theta_{1}\left(\theta_{1}+\theta_{2}\right)\right]
$$


The structure of a pure hard diatomic near a hard wall is usually determined as the limiting case of a pair of hard dumbbell/hard sphere (with an infinitely large radius and concentration $\approx 0$ ). To determine the distribution function within our approach we consider instead of (quantities of) the infinitely large hard sphere a hard disk of the radius $r_{d}$ (see Figure 2a for the perpendicular orientation of ehd with respect to the wall), with the zero volume and zero mean radius and with the surface area equal to $S_{d}=\pi r_{d}^{2}$. The geometric quantities of the combined body possess then following forms

$$
\begin{aligned}
& S^{C B} / \pi \sigma_{1}^{2}=S_{1} / \pi \sigma_{1}^{2}-\frac{1}{2}\left[(1-\sin \theta)-r_{p}^{*}\left(1+r_{p}^{*}\right)(\pi / 2+\theta) \cos \theta+r_{p}^{* 2}(1+\sin \theta)\right] \\
& 2 R^{C B} / \sigma_{1}=R_{1} / r_{1}-\frac{1}{2}\left[(1-\sin \theta)+\left(1+r_{p}^{*}\right)(\pi / 2+\theta) \cos \theta / 2-r_{p}^{*}(1+\sin \theta)\right]
\end{aligned}
$$

and

$$
\begin{aligned}
& V^{C B} /\left(\pi \sigma_{1}^{3} / 6\right)=V_{1} /\left(\pi \sigma_{1}^{3} / 6\right)-\frac{1}{2}\left(1-\sin \theta-\cos ^{2} \theta \sin \theta / 2\right) \\
& +\frac{3}{4} r_{p}^{*}\left(1+r_{p}^{*}\right) \cos \theta\left[\left(1+r_{p}^{*}\right) \cos \theta(1+\sin \theta)-r_{p}^{*} \cos \theta \sin \theta\right. \\
& \left.-r_{p}^{* 2}\left(1+r_{p}^{*}\right)(\pi / 2+\theta) \cos \theta\right]+\frac{r_{p}^{* 3}}{2}\left(\cos ^{2} \theta \sin \theta+2 \sin \theta+2\right)
\end{aligned}
$$

Here $\theta$ is an angle defined as $\theta=\arcsin \left(2 z /\left(1+r_{p}^{*}\right)-1\right)$ and $z$ is the reduced distance (related to $\sigma_{1}$ ) of the site from the disk.

For distances $z>\left[r_{p}^{*}+\sqrt{(}\left(1+2 r_{p}^{*}\right)\right] / 2$ the combined body decomposes into two parts ending in cusps, see Figure $2 \mathrm{~b}$, and the integrals which determine the contribution of the saddle parts to the $\mathrm{CB}$ surface area or volume have to be considered for bounds $\phi-\theta$, [where $r_{p}^{*} \cos \phi=\left(1+r_{p}^{*}\right) \cos \theta$ ]. Thus, one has to subtract contributions for angles $(-\phi, \phi)$ - i.e. $\delta R, \delta S, \delta V$, from the above expressions. These contributions are

$$
\begin{gathered}
\delta R / r_{1}=-\phi\left(1+r_{p}^{*}\right) \cos \theta / 2+r_{p}^{*} \sin \phi \\
\delta S / \pi \sigma_{1}^{2}=\phi r_{p}^{*}\left(1+r_{p}^{*}\right) \cos \theta-r_{p}^{* 2} \sin \theta \\
\delta V /\left(\pi \sigma_{1}^{3} / 6\right)=\frac{1}{2}\left[3 r_{p}^{*}\left(1+r_{p}^{*}\right)^{2} \cos ^{2} \theta \sin \phi\right. \\
\left.-3 r_{p}^{* 2}\left(1+r_{p}^{*}\right) \cos \theta(\cos \phi \sin \phi+\phi)+r_{p}^{* 3} \sin \phi\left(\cos ^{2} \phi+2\right)\right]
\end{gathered}
$$

The above expressions are valid when the structure of the larger site/wall 
is considered. In the case of smaller site/wall functions, the probe sphere for small values of distance $z$ contact - in special orientations, see Figure 3 - the larger instead of the smaller site. Then in eqs (9)-(11) it is necessary to consider the reduced quantities of the larger site, equal in all the cases to one [instead of $2 R_{1} / \sigma_{1}, S_{1} /\left(\pi \sigma_{1}^{2}\right)$ and $V_{1} /\left(\pi \sigma_{1}^{3} / 6\right)$ ].

Functional $Q^{(C B)}$ is approximated as

$$
Q^{(C B)}=S^{(C B)}\left(4 \pi R^{(C B) 2} / S^{(C B)}\right)^{1 / 4}
$$

\subsection{Mixtures}

In this paper we study a special case of hard body mixtures, namely mixtures of the heteronuclear hard dumbbells and hard spheres (whose diameter is equal to the diameter of the larger hhb site, $\sigma_{h s}=\sigma_{1}$ ).

The hard body/wall correlation function of a non-spherical particle can be evaluated from eqs (9)-(11)which are applicable with the following changes: (i) the reference quantities $R_{r e f}, S_{r e f}, V_{r e f}, Q_{r e f}$ for a binary mixtures are defined as $X_{\text {ref }}=\Sigma x_{i} X_{i}$ (where $x_{i}$ denotes the mole fraction and $\mathrm{X}$ a single geometric quantity). (ii) The non-sphericity parameters $\alpha, \beta$ in the above equations (for pure fluid systems) should be replaced by parameters $\alpha_{s}, \beta_{s}$ defined in eq (2). (iii) the reduced probe radius $r_{p}^{*}$ is determined as a weighted average from those of single components (see the discussion in Ref[21]).

It is obvious that in the case of hard sphere/hard wall correlation function, one has to consider instead of $R_{1} /\left(\sigma_{1} / 2\right), S_{1} / \pi \sigma_{1}^{2}, V_{1} /\left(\pi \sigma^{3} / 6\right)$ and $Q_{1} /\left(\sigma_{1} / 2\right)^{2}$ in our case the hs values $R_{2} /\left(\sigma_{1} / 2\right)=S_{2} / \pi \sigma_{1}^{2}=V_{2} /\left(\pi \sigma_{1}^{3} / 6\right)=$ $Q_{2} /\left(\sigma_{1} / 2\right)^{2}=1$.

\section{Results and discussion}

Firstly we consider a pure heteronuclear hard dumbbell (hhd) system. The studied dumbbell is characterized by the reduced site-site distance $L^{*}=$ $l / \sigma_{1}=0.625$ and the ratio of the site diameters $p=\sigma_{2} / \sigma_{1}=0.5$. Because of the low p-value, the perpendicular orientation of hhd to the hard wall is decisive for determination of the correlation function of the larger site. For this orientation the background correlation function is evaluated first, and consequently multiplied by the probability $\mathcal{P}$ of the general orientation of the dumbbell axis with respect to the normal of the wall. It is evident that for the given distance of the larger site from the wall, $z$, orientations with angles $\zeta>\pi / 2+\arcsin \left(\left(z-r_{2}\right) / L^{*}\right)$ are forbidden. Thus, in the simplest approach we take as the probability function the ratio of acceptable to all 
possible orientation. For $z \geq z_{c}=p / 2+L^{*}$ the probability is equal to one; at values $z=z_{c}$ the correlation function discloses a cusp.

The calculated correlation functions for packing fractions $y=0.407$ and 0.356 are compared with simulation data of Nezbeda et al. [23] in Figure 4. It is obvious that the geometric method yields a fair prediction of the correlation function for both the studied systems with exception of the distances close to the contact value (where, however, experimental values taken from figures in $\operatorname{Ref}[23]$ are rather uncertain).

Evaluation of the background correlation function of the smaller site of the considered dumbbell (with diameter $\sigma_{2}=0.5 \sigma_{1}$ )/wall in the perpendicular orientation is more complex, because for the lowest $z$ values, the probe sphere with $r_{p} / r_{1}=0.9$ (i.e. only slightly smaller than the larger site of hhd) contacts the larger site instead of the smaller site situated closer to the wall; for parallel orientation and majority of general orientations the combined body is determined mainly by the site - probe contacts. To this end, we evaluate bcf's for the larger site/wall situation, determined, however, for wall-site distance $z+d_{a v}$ with the average distance $d_{a v}$ resulting from $d_{\max }=L^{*}$ and that of a minimum, where the larger site is in contact with the wall. To get distribution function $g_{W H D 2}$, we multiply the obtained bcf-value by the probability ratio of allowed and total orientations. Dependence of the distribution function of the smaller hhd site/wall on the distance $z$, of the center of site 2 from the wall is compared with the experimental data on the most important interval of $z$ in Figure 5. Larger differences, observed in the central part of the graph are due to neglecting the smaller site-probe contributions to the combined body.

Next we consider approximately equimolar mixtures of hhd(1) and hard sphere(2) (hs) near a hard wall. To determine the hs/wall correlation function we calculate bcf; all the reduced geometric quantities of HS are equal to one, however, the reference quantities are given by prescription $X=\sum x_{i} X_{i}$ and instead of $\alpha \alpha_{s}$ has to be used. The reduced probe radius $r_{p} / r_{1}$ is here $r_{p}^{*}=0.95$. For the hs/wall function, there are no forbidden orientations, therefore $g_{W H S}=Y$. Dependence of $g_{W H S}$ on the reduced distance $z$ for mixtures with the packing fraction $y=0.407$ and composition $x_{1}=0.514$ and $y=0.306, x_{1}=0.523$ are compared with values taken from a figure in Ref [23]. Figure 6 shows a fair agreement of theory with simulation results. Evaluation of the correlation function $g_{W H D 1}$ resembles much the approach described in the previous part for pure hhd, however, with values of $r_{p}^{*} \alpha_{s}, \beta_{s}$ and $R_{r e f}, S_{r e f}, V_{r e f}, Q_{r e f}$ valid for the mixture and used here for in the case of 
the hhd + hs solution. The correlation function $g_{W H D 1}$ was again obtained by multiplying bcf for the given distance $z$ by the probability function (same as in the case of pure hhd). Theoretical correlation functions calculated for the packing fractions and compositions mentioned above, agree well with experimental results; at $z=0.875$ cusps are found, corresponding to a transition from the restricted to free rotation, see Figure 7.

Determination of the distribution function of the smaller site / hard wall in the mixture of hhd + hs follows the way used in the previous case, however, the combined body of the hhd+hard wall is taken as a result of contact between larger site(1) and the probe hard sphere, as in the case of pure hhd fluid. Comparison of the distribution function of smaller site/hard wall in the hhd + hs mixture with the packing fraction $\mathrm{y}=0.358$, and the mole fraction $x_{1}=0.525$ with simulation results are shown in Figure 8 for the interval of reduced distances $z \in(0.25,1)$. It is apparent that the effect of hard sphere (in mixture) on the course of the theoretical $g_{W H D 2}$ is small and the distribution function resembles that for pure hhd fluid; the same is true for experimental dependence of $g$ vs $z$ for a mixture and pure fluid.

\section{Conclusions}

The present study of heteronuclear hard dumbbell with the considerably different site diameters and relatively short reduced site-site distance brings in comparison with previously considered homonuclear dumbbell (Ref[20]) some simplifications and some new problems. Simplifications consist in fact that the distribution function determined on the basis of background correlation function for the perpendicular orientation of hhd with respect to the wall, multiplied by the probability of different general orientations is more accurate than in the case of homonuclear hard dumbbells. This is a result of the smaller effect of the other site on the range of acceptable orientations. On the other hand, determination of the the smaller site/hard wall bcf faces difficulties, as in the case of the perpendicular orientation and shortest distances $z$, the probe sphere (with $r_{p} \approx r_{1}$ ) contacts firstly the more removed larger site and only for larger distances $z$ it contacts the smaller site, situated near the wall. For general orientations, interactions of the probe with the larger sphere of hhd are more important. Therefore we employ for the determination of the geometric quantities of the combined body the bcf for the removed larger site+hard probe situation. This fact causes some uncertainties within the procedure of determining the distribution function. 
In this and previous contributions we tried to show the potential of the simple method to determine distribution functions in inhomogeneous systems (on the basis of residual chemical potential of the hard fluid molecules, of the hard wall and of the combined body). For hard dumbbells and other non-spherical models, an improved but more involved version can be worked out, where the geometric quantities of the combined body for the single orientations are determined for each distance $z$ and some averaging procedure to get the average site-wall distribution functions is applied.

\section{Acknowledgement}

The work has been financially supported by grant No. IAA 400720710 of the Grant Agency of the Academy of Sciences of the Czech Republic.

\section{References}

[1] J. R. Henderson and F. Van Swol, Molec. Phys. 51, 991 (1984).

[2] R. Pospíšil, A. Malijevský, P. Jech and W. R. Smith, Molec. Phys. 78, 1461 (1993).

[3] S. Labík and W. R. Smith, Molec. Phys. 88, 1411 (1996).

[4] W. R. Smith and H. L. Vörtler, Molec. Phys. 101, 805 (2003).

[5] B. K. Peterson and K. E. Gubbins, Molec. Phys. 62, 215 (1987).

[6] J. R. Henderson, Molec. Phys. 59, 89 (1986).

[7] Y. Q. Zhou and G. Stell, Molec. Phys. 66, 767 (1989).

[8] I. K. Snook and D. Henderson, J. Chem. Phys, 68, 2134 (1978).

[9] Y. Rosenfeld and P. Tarazona, Molec. Phys. 95, 141 (1998).

[10] D.M. Kroll and B. B Laird, Phys Rev. A: At., Mol., Opt. Phys. 44, 4806 (1990). 
[11] A. Malijevský, J. Chem. Phys. 125, 194519 (2006).

[12] E. Meeron and A.J.F. Siegert, J. Chem. Phys. 48, 3139 (1968).

[13] J. A. Ballance and R. J. Speedy, Molec. Phys. 54, 1035 (1985).

[14] R. J. Speedy, J. Chem. Soc. Faraday Trans.II, 73, 714 (1977).

[15] S. Labík, A. Malijevský and I. Nezbeda, Molec. Phys., 60, 1107 (1987).

[16] S. Labík, A. Malijevský and W. R. Smith, Molec. Phys., 83, 983 (1994).

[17] T. Boublík, Molec. Phys. 59, 371 (1986).

[18] T. Boublík, Molec. Phys. 91, 161 (1997).

[19] T. Boublík, Molec. Phys. 104, 3425 (2006).

[20] T. Boublík, J. Phys. Chem. C 111, 15505 (2007).

[21] T. Boublík, Collect. Czech. Chem. Commun. 73, 388 (2008 ).

[22] T. Boublík, J. Phys. Chem. B, in press.

[23] I. Nezbeda, M. R. Reddy and W.R. Smith, Molec. Phys. 71, 915 (1990).

[24] D. Henderson, S. Sokolowski and D. Wasan, Molec. Phys. 93, 295 (1998).

[25] T. Boublík, Molec. Phys. 100, 3443 (2002).

[26] M. L. Connolly, J. Appl. Crystallogr. 16, 548 (1983).

[27] M. L. Connolly, J. Am. Chem. Soc. 107, 1118 (1985). 


\section{Captions}

Figure 1. Geometry of the heteronuclear hard dumbbell of the reduced sitesite length $L$ and hard sphere radii $r_{1}, r_{2}$ and the probe radius $r_{p}\left(L^{*}=\right.$ $\left.0.625, \sigma_{2} / \sigma_{1}=0.5\right)$.

Figure 2. Geometry of the hard dumbbell/hard wall in the perpendicular orientation. $z$-dumbbell-site reduced distance, $\theta_{i}$ - characteristic angle, $r_{p}$ probe sphere radius, a) for shorter distances, b) for $z>\left[r_{p}+\left(1+2 r_{p}\right)^{1 / 2}\right] / 2$.

Figure 3. The same as in Figure 2 for the smaller site.

Figure 4. Larger site/wall distribution function for packing fractions $y=$ 0.407 (o, full line) and $y=0.356(\Delta$, dash line).

Figure 5. Smaller site/wall distribution function for the system with the packing fraction $y=0.356$.

Figure 6. Approximately equimolar mixture of hard dumbbell+hard sphere. hard spher/wall distribution function, $y=0.407, x_{1}=0.514$, (o, full line), $y=0.306, x_{1}=0.523(\Delta$, dash line $)$.

Figure 7. The same as in Figure 6 but for the site/wall distribution function.

Figure 8. Smaller site/wall distribution function for the hhd-hs mixture $\left(y=0.358, x_{1}=0.525\right)$ 


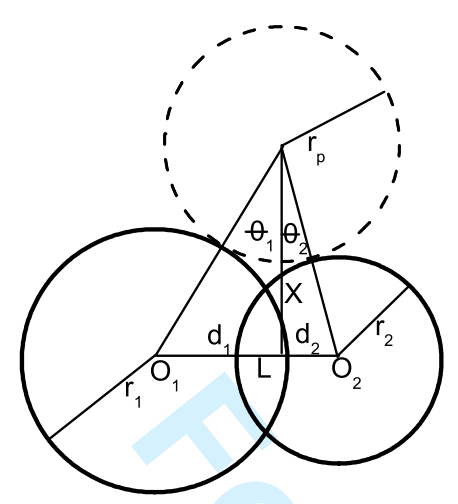

Fig.1. 
1

2

3

4

5

6

10

11

12

13

14

15

16

17

18

19

20

21

22

23

24

25

26

27

28

29

30

31

32

33

34

35

36

37

38

39

40

41

42

43

44

45

46

47

48

49

50

51

52

53

54

55

56

57

58

59

60
Fig.2.

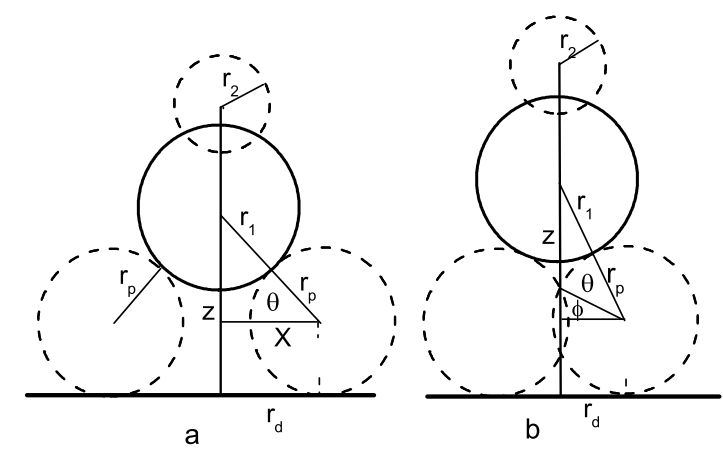

16

URL: http://mc.manuscriptcentral.com/tandf/tmph 


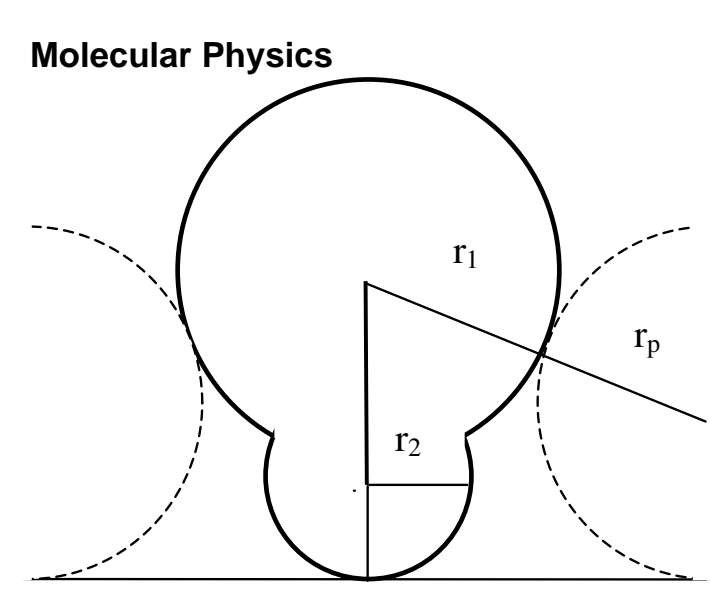


1

2

3

4

5

6

7

8

9

10

11

12

13

14

15

16

17

18

19

20

21

22

23

24

25

26

27

28

29

30

31

32

33

34

35

36

37

38

39

40

41

42

43

44

45

46

47

48

49

50

51

52

53

54

55

56

57

58

59

60

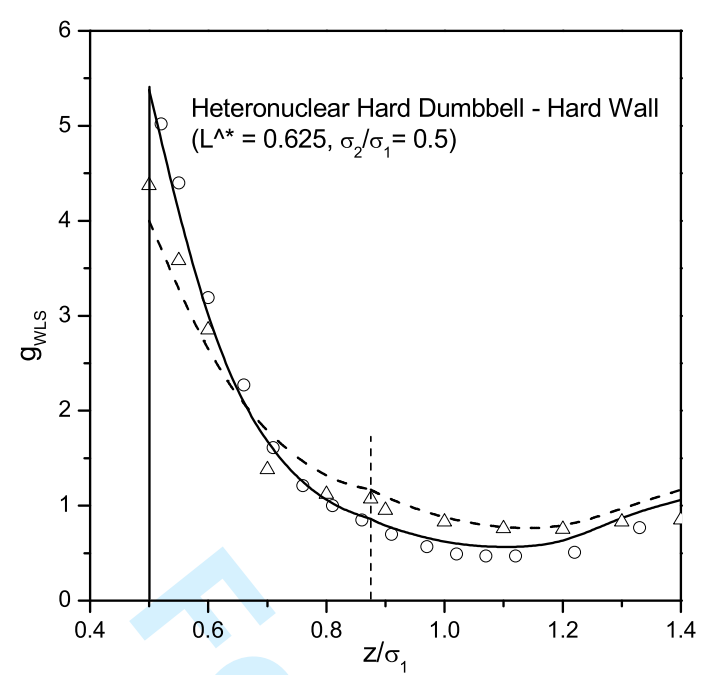

Fig.4. 


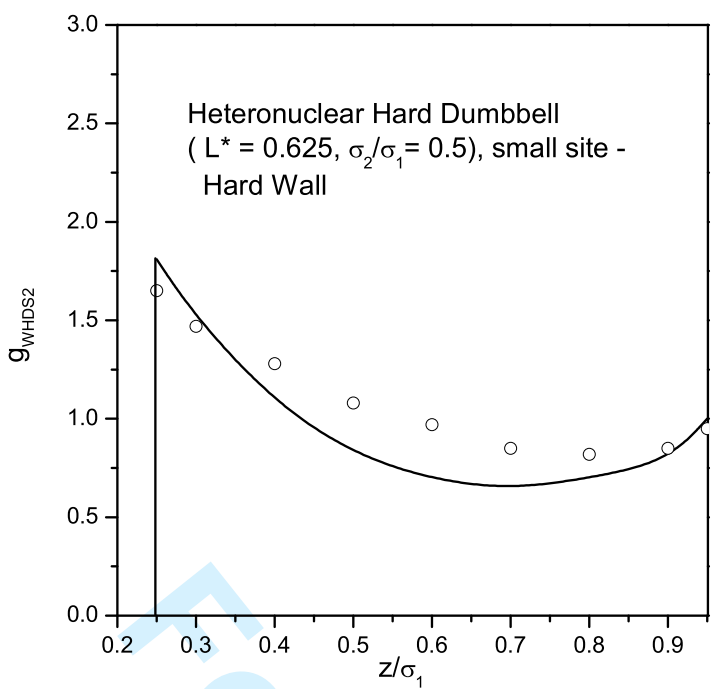

Fig. 5 
1

2

3

4

5

6

7

8

9

10

11

12

13

14

15

16

17

18

19

20

21

22

23

24

25

26

27

28

29

30

31

32

33

34

35

36

37

38

39

40

41

42

43

44

45

46

47

48

49

50

51

52

53

54

55

56

57

58

59

60

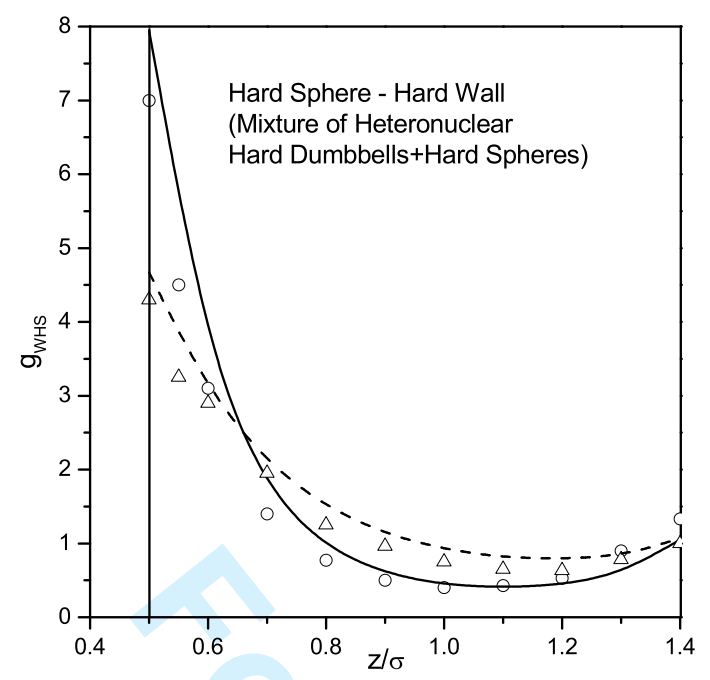

Fig.6. 


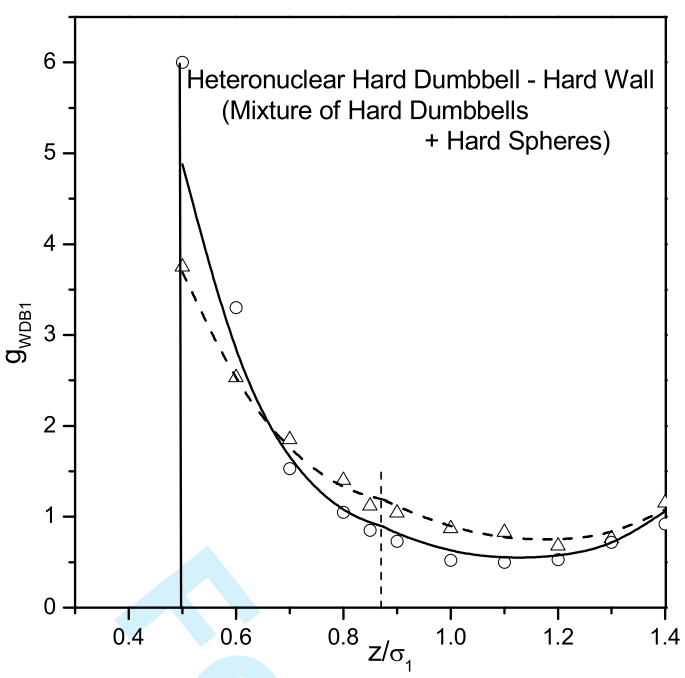

Fig.7. 
1

2

3

4

5

6

7

8

9

10

11

12

13

14

15

16

17

18

19

20

21

22

23

24

25

26

27

28

29

30

31

32

33

34

35

36

37

38

39

40

41

42

43

44

45

46

47

48

49

50

51

52

53

54

55

56

57

58

59

60

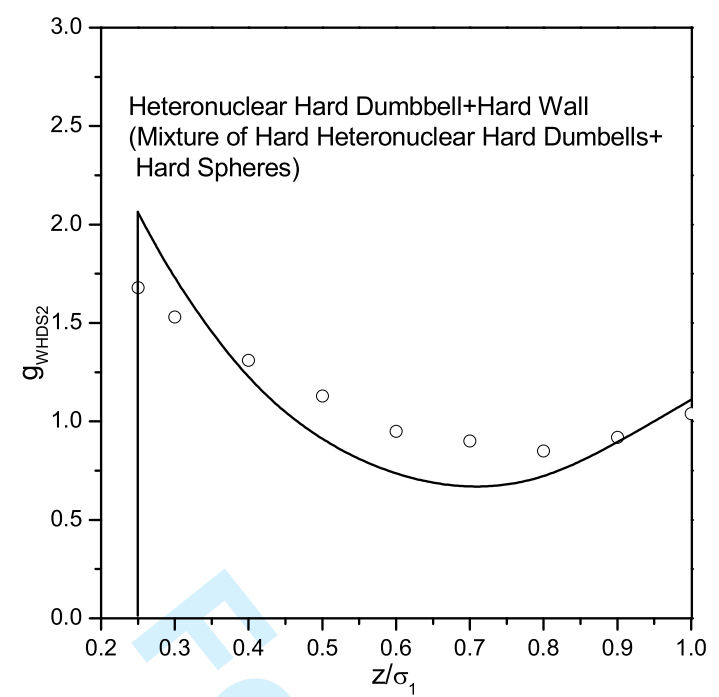

Fig.8 


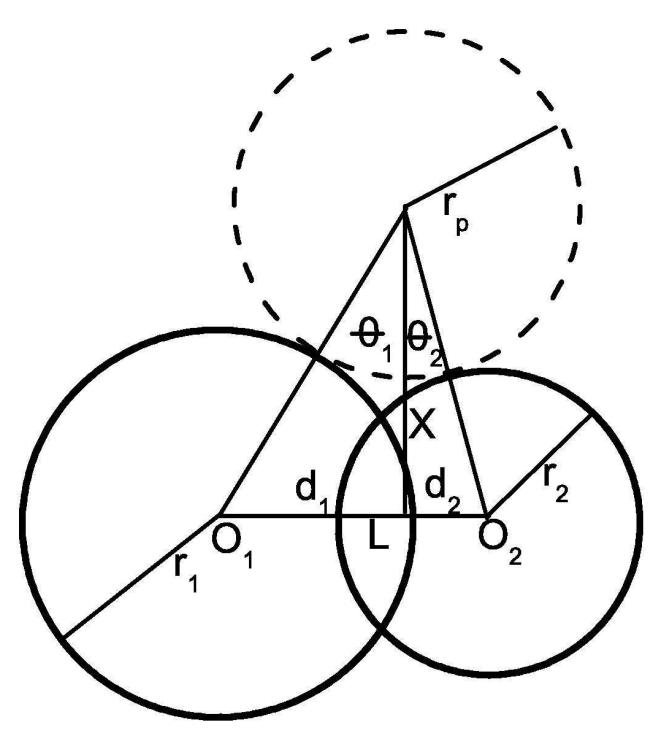

$89 \times 73 \mathrm{~mm}(600 \times 600$ DPI $)$ 


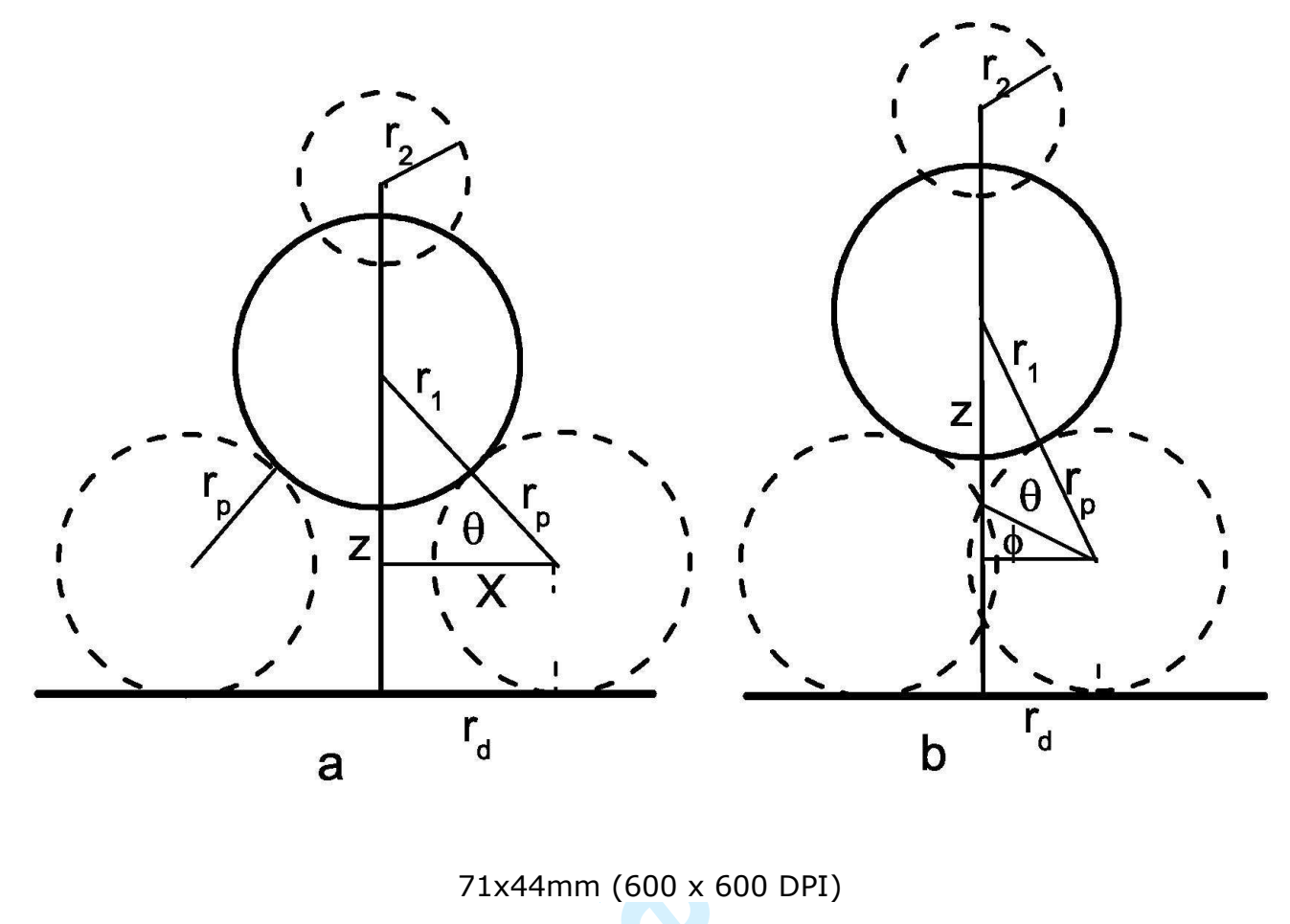

URL: http://mc.manuscriptcentral.com/tandf/tmph 
Molecular Physics

Page 26 of 30

1
2
3
4
5
6
7
8
9
10
11
12
13
14
15
16
17
18
19
20
21
22
23
24
25
26
27
28
29
30
31
32
33
34
35
36
37
38
39
40
41
42
43
44
45
46
47
48
49
50
51
52
53
54
55
57
58

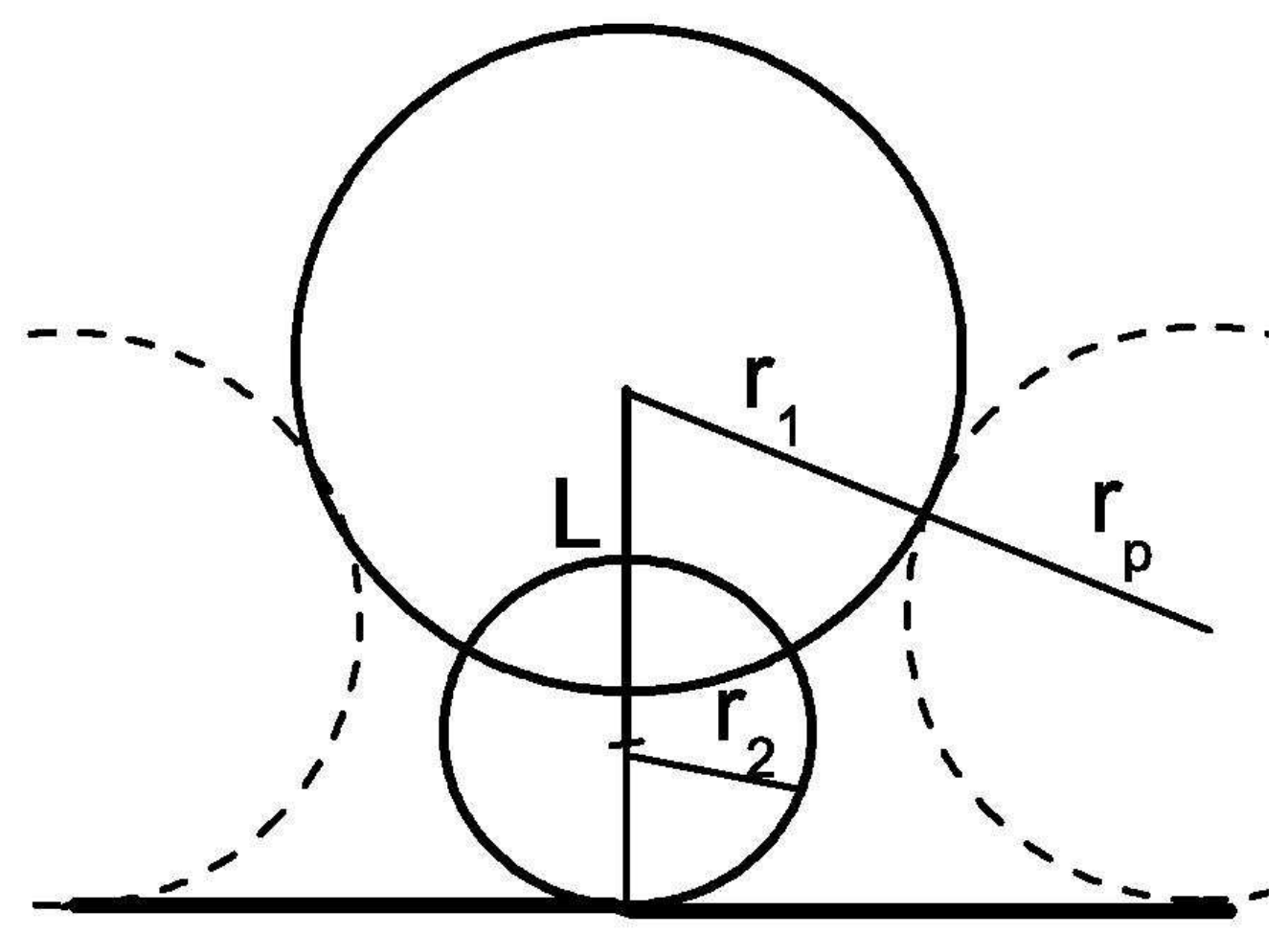

$37 \times 37 \mathrm{~mm}(600 \times 600 \mathrm{DPI})$

URL: http://mc.manuscriptcentral.com/tandf/tmph 


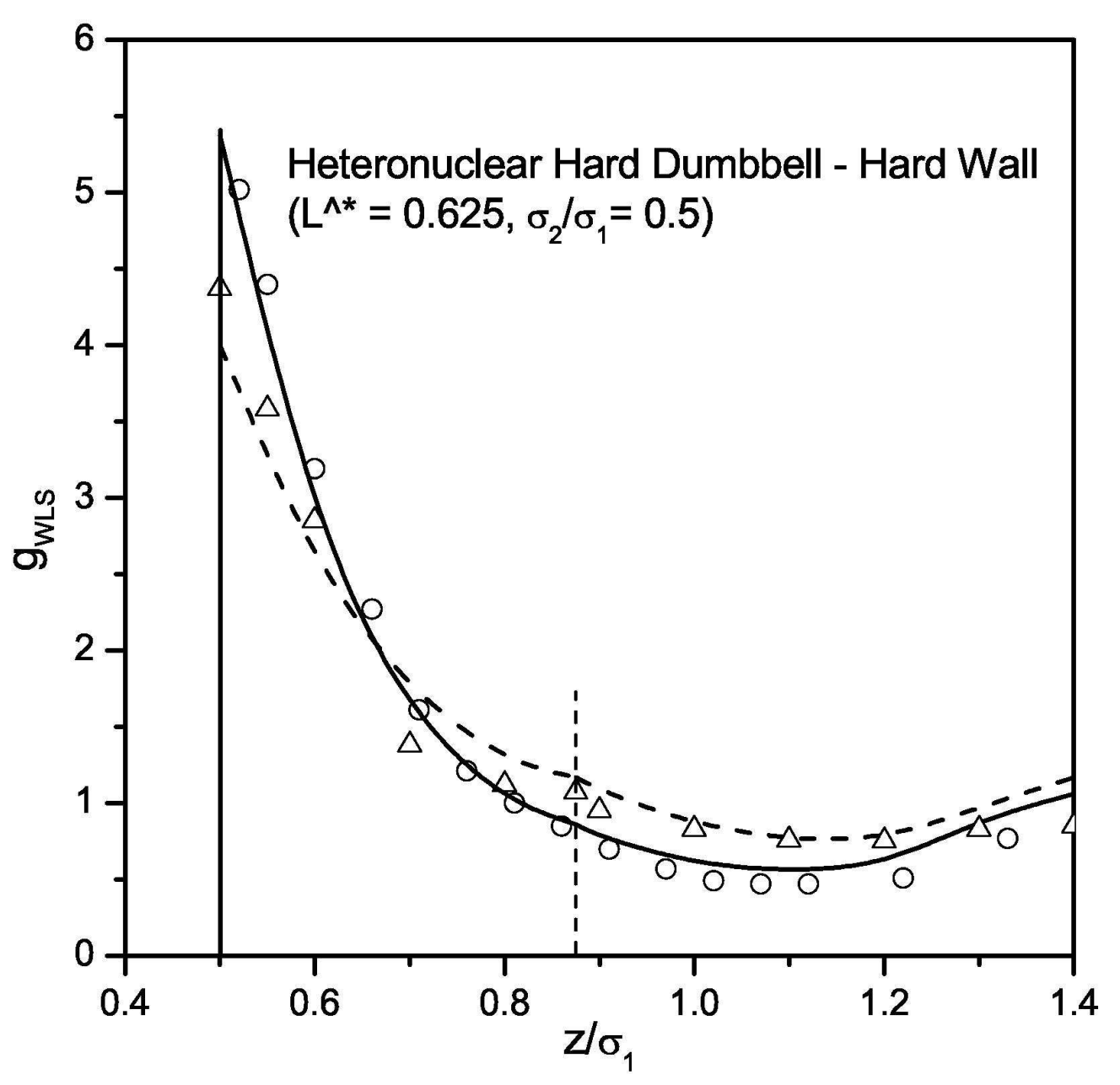

$69 \times 66 \mathrm{~mm}(600 \times 600 \mathrm{DPI})$ 


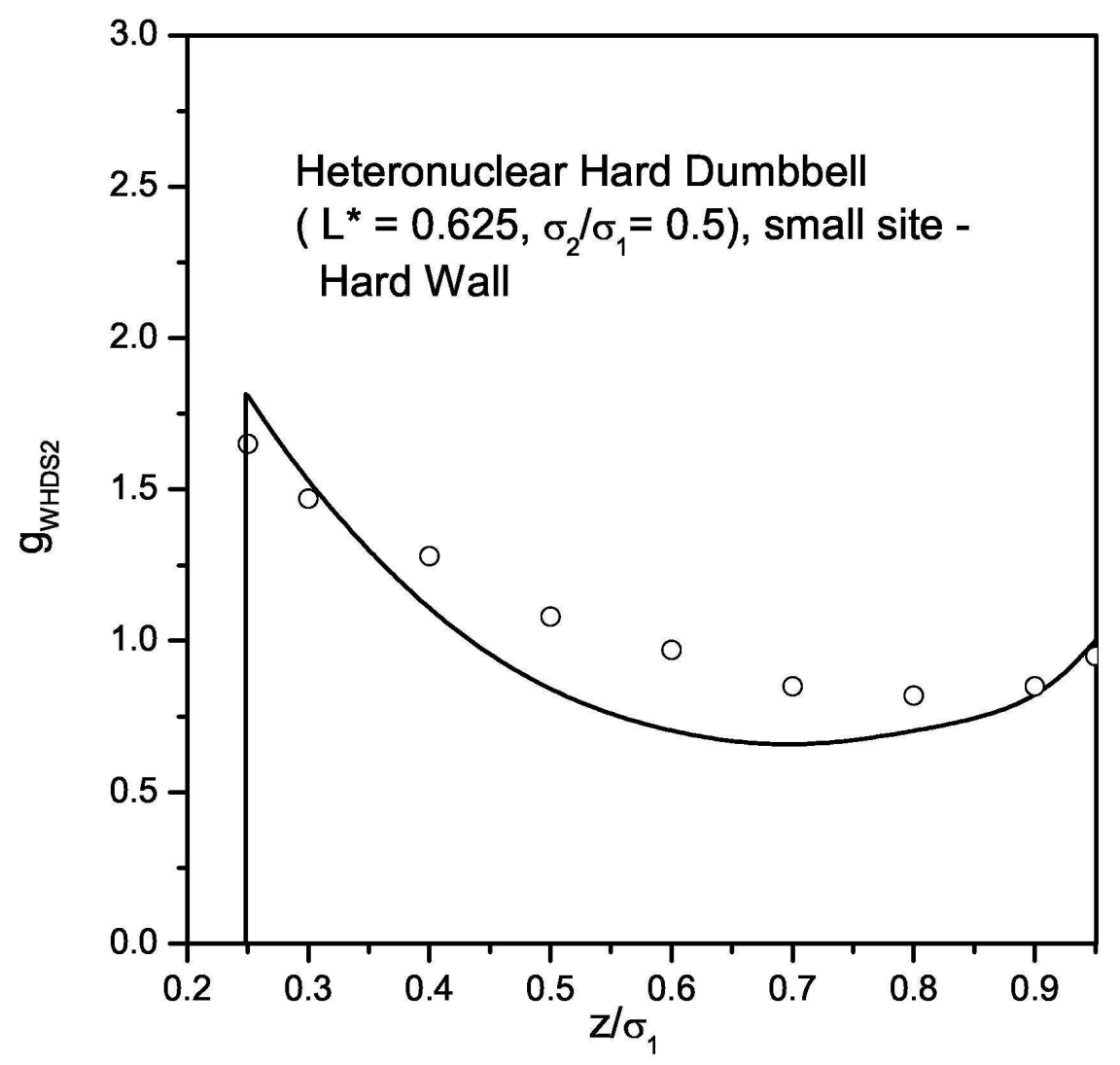

$83 \times 81 \mathrm{~mm}(600 \times 600 \mathrm{DPI})$

URL: http://mc.manuscriptcentral.com/tandf/tmph 


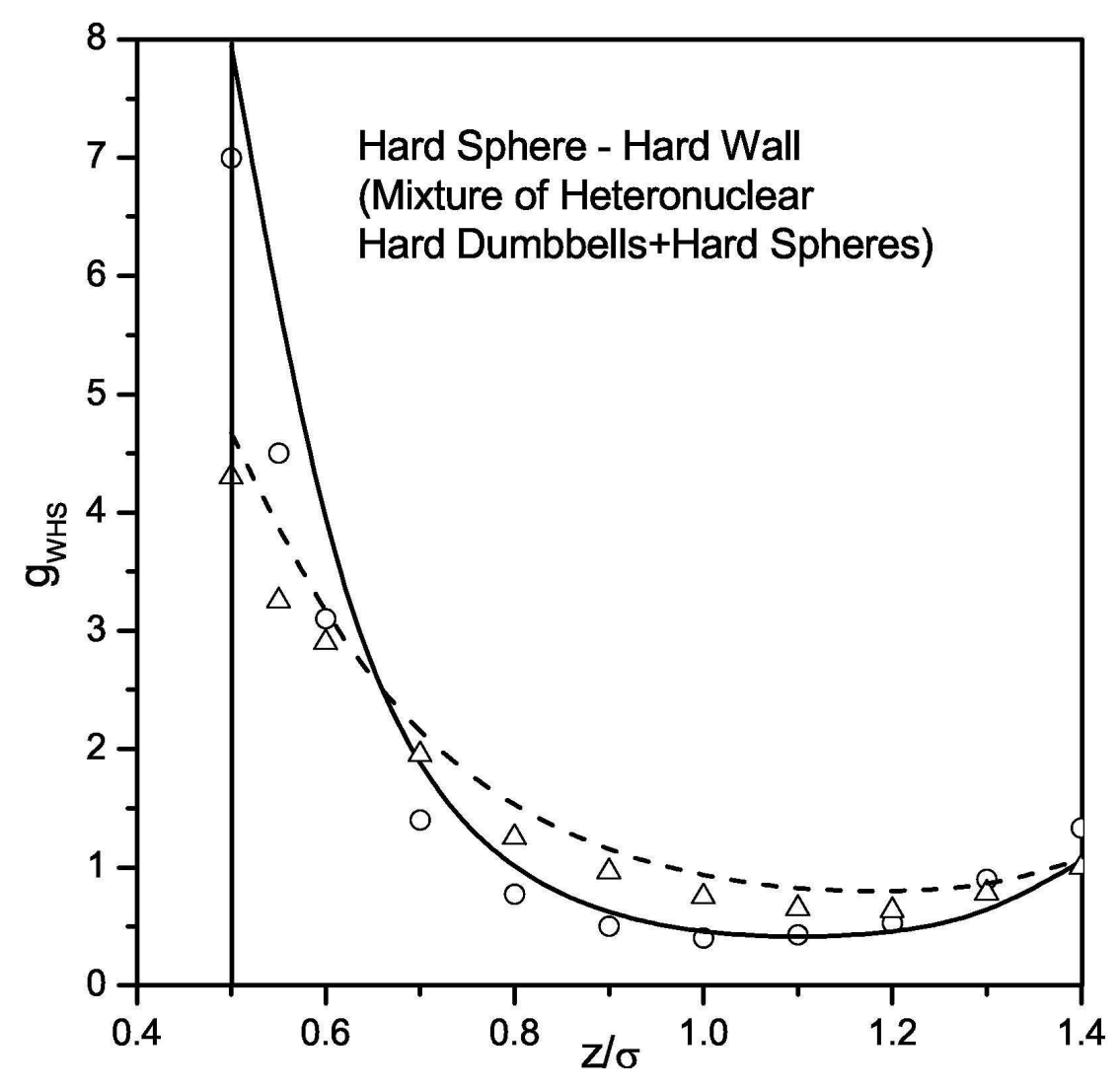

$81 \times 79 m m(600 \times 600$ DPI $)$ 


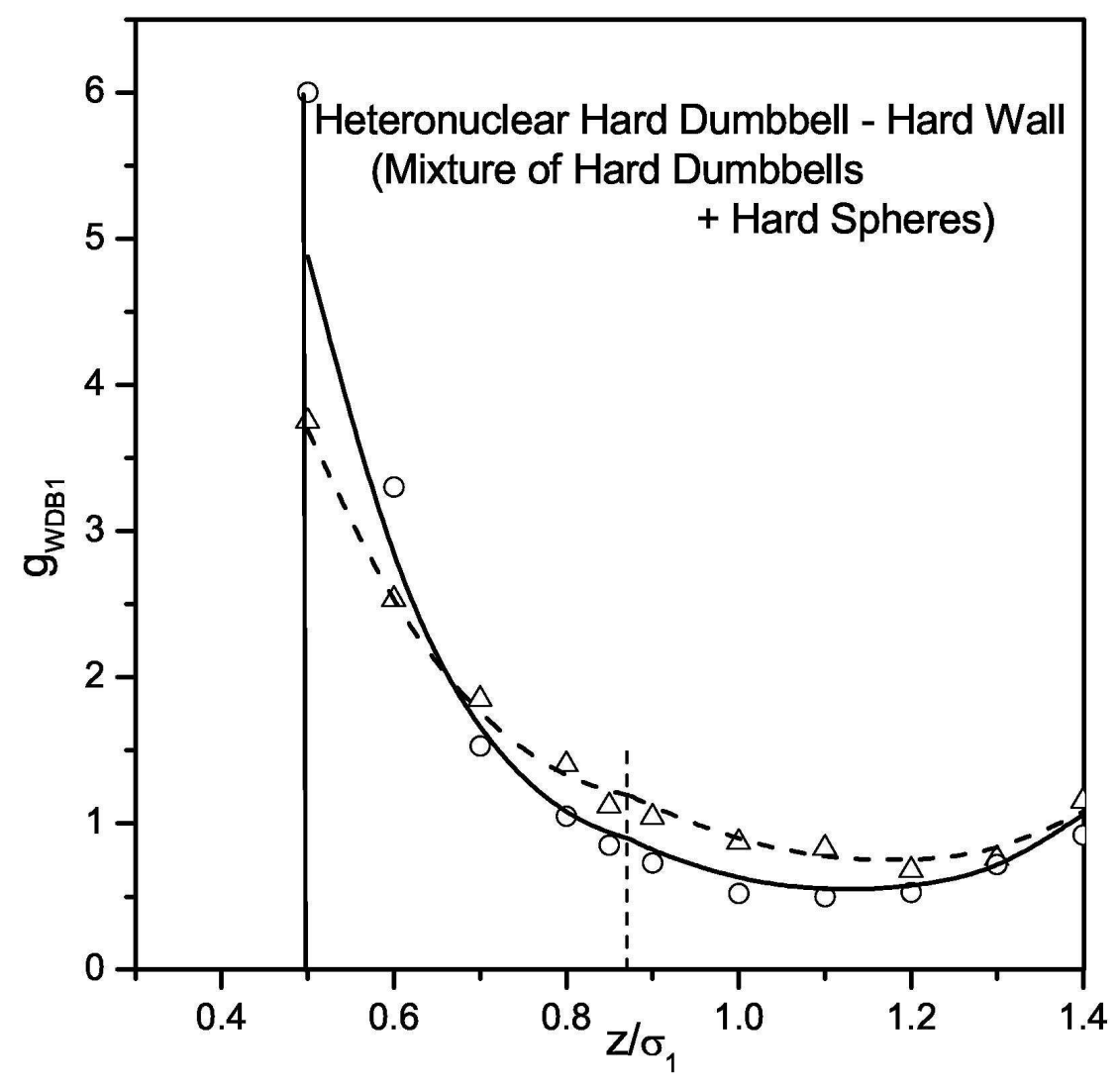

$81 \times 78 \mathrm{~mm}(600 \times 600$ DPI $)$ 


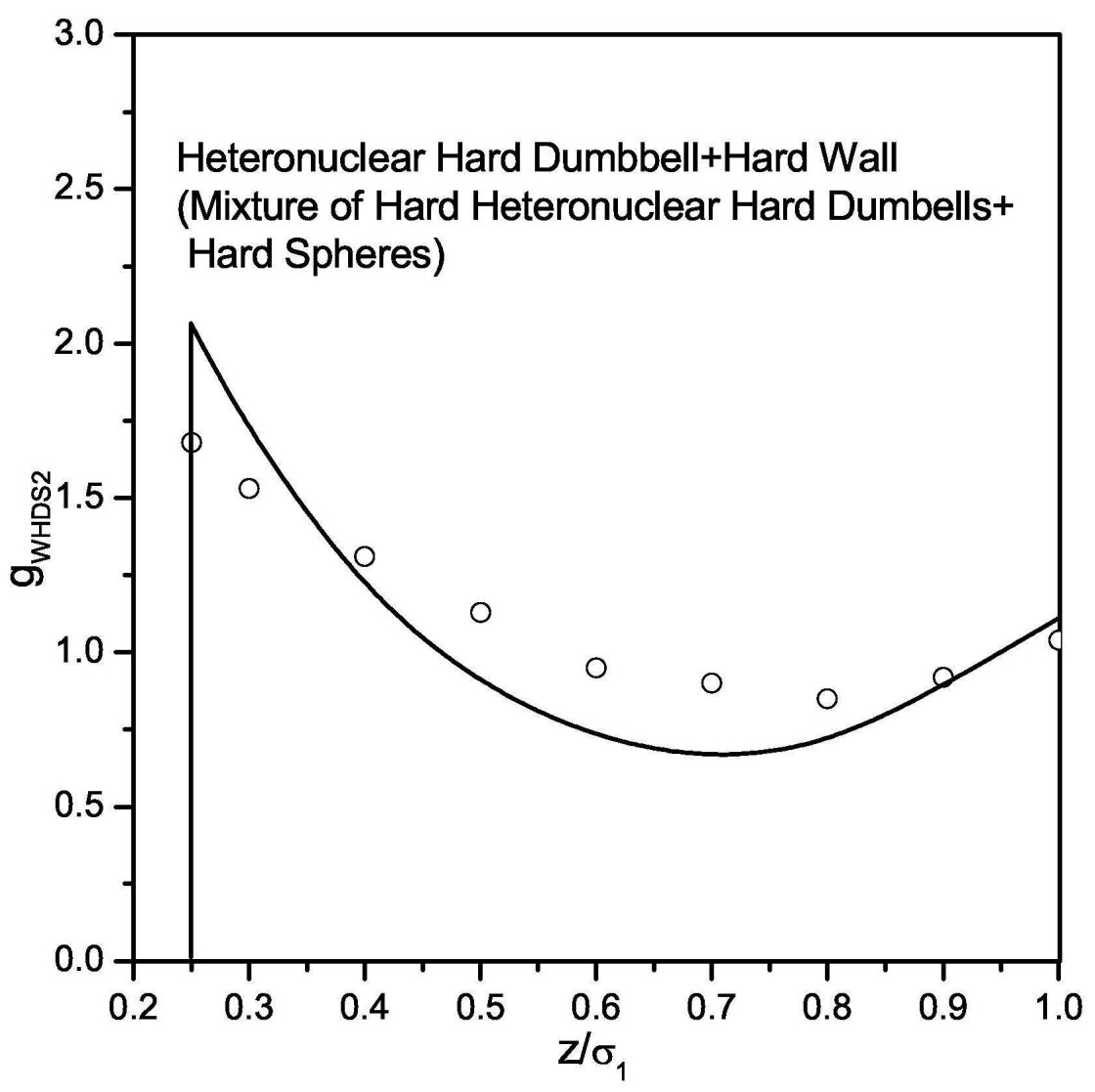

$82 \times 81 \mathrm{~mm}(600 \times 600 \mathrm{DPI})$ 